This is an Accepted Manuscript of an article published by Taylor \& Francis in 'Archives of Suicide Research' on 2020-11-01, available online: https://www.tandfonline.com/10.1080/13811118.2020.1841052.

\title{
Evaluating the Effectiveness of a Brief Mindfulness Activity in University Students With Non-Suicidal Self-Injury Engagement
}

\begin{abstract}
Current theoretical frameworks posit that engagement in non-suicidal self-injury (NSSI) is due to an inability to regulate one's emotions. In turn, mindfulness-based interventions have been shown to enhance emotion regulatory processes in those who engage in NSSI

Objective. The purpose of the present study was to determine whether a brief mindfulness activity was differentially effective at increasing state mindfulness and decreasing stress following a stress induction task in university students with versus without a history of NSSI engagement.
\end{abstract}

Method. The sample consisted of two groups of participants who identified as women: participants with a history of NSSI engagement (NSSI; $\left.n=57 ; M_{\mathrm{age}}=20.09, S D=2.05\right)$ and participants without (no-NSSI; $n=87 ; M_{\text {age }}=20.22, S D=1.94$ ). All participants were asked to complete pre-intervention measures of state mindfulness and stress and were randomly assigned to either a mindfulness activity (body scan) or control task condition. Following the completion of their respective activities, a Stroop stress induction task was conducted and participants completed post-intervention measures of state mindfulness and stress.

Results. Two 3-way mixed ANOVAs (Time X NSSI status X Condition) were conducted and revealed significant time by condition interactions for both state mindfulness, Wilk's $\Lambda=.93$, $F(1,140)=10.70, p=.001, \eta_{p}^{2}=.07$, and stress, Wilk's $\Lambda=.97, F(1,140)=4.21, p=.04, \eta_{p}^{2}=$ .03. As such, both groups (NSSI/no-NSSI) demonstrated similar increases in state mindfulness and decreases in stress in response to the brief mindfulness activity following the stress induction. Implications for future research and practice will be discussed. 
Keywords: mindfulness, non-suicidal self-injury, university, emotion regulation

\section{INTRODUCTION}

Non-suicidal self-injury (NSSI) is defined as the deliberate and intentional destruction of one's own bodily tissue without suicidal intent and for reasons that are not socially sanctioned (e.g., tattoos and body piercings; International Society for the Study of Self-Injury, 2007). Common NSSI behaviors include cutting, scratching, burning of skin, as well as hitting one's self (Klonsky \& Muehlenkamp, 2007). NSSI engagement is associated with mental health difficulties and other unhealthy coping behaviors (e.g., Hilt, Nock, Lloyd-Richardson, \& Prinstein, 2008) as well as a seven-fold increase in the likelihood of attempting suicide (e.g., Deliberto \& Nock, 2008; Hasking, Momeni, Swannell, \& Chia, 2008). NSSI is commonly reported as a means to cope with stress or negative feelings (Klonsky, 2007) and, like healthy coping behaviors, is effective at alleviating experienced distress, which further reinforces its use (Nock \& Prinstein, 2004). Research has shown that those who engage in NSSI report higher levels of daily stress (Kiekens et al., 2015), and indications that those who engage in NSSI may be hyper- reactive to stress (Fliege et al., 2006; Reichl et al., 2016).

NSSI is commonly reported among university students with between 15 to $20 \%$ reporting lifetime engagement (Swannell, Martin, Page, Hasking, \& St John, 2014), and between 6 to 8\% reporting recent engagement in the behavior (i.e., sometime during the last 12 months; Whitlock et al., 2011). Additionally, a recent review by Cipriano et al. (2017) found that prevalence rates of this behavior in university students is higher than other groups. Unsurprisingly, engagement in NSSI often appears concurrently with depression and anxiety among university students (Serras, Saules, Cranford, \& Eisenberg, 2010) and those who engage in NSSI persistently through their studies report greater psychosocial risk (Hamza \& Willoughby, 2014). As such, there is a need to 
This is an Accepted Manuscript of an article published by Taylor \& Francis in 'Archives of Suicide Research' on 2020-11-01, available online: https:/www.tandfonline.com/10.1080/13811118.2020.1841052.

identify programs that support this subpopulation of students during an already emotionally stressful period in their lives.

Mindfulness has attracted a considerable amount of attention in western societies (Tanay \& Bernstein, 2013). Although definitions vary (e.g., Nilsson \& Kazemi, 2016), mindfulness is commonly defined as paying attention on purpose to the present moment with nonjudgmental acceptance (Kabat-Zinn, 1994). It has been conceptualized as either a disposition or a state. Dispositional mindfulness is defined as one's tendency to be mindful in their daily life. According to Brown and Ryan (2003), without inter- vention, dispositional mindfulness is believed to be stable over time and has been asso- ciated with lower levels of stress, anxiety, and depression (Brown \& Ryan, 2003; Cash \& Whittingham, 2010; Segal, Teasdale, Williams, \& Gemar, 2002) as well as with improve- ments in emotion regulation and emotional reactivity (Creswell \& Lindsay, 2014; Hayes \& Feldman, 2004).

On the other hand, state mindfulness is dependent on context and can vary moment- tomoment (Bishop et al., 2006). When practicing mindfulness, state mindfulness levels increase (e.g., Lau et al., 2006) which has been found to lead to changes in dispositional mindfulness overtime (Kiken, Garland, Bluth, Palsson, \& Gaylord, 2015). In other words, through practice, state mindfulness is accumulated, which leads to increases in dispositional mindfulness and its associated benefits such as reduced psychological dis- tress (Kiken et al., 2015). State mindfulness is an important concept because it demon- strates that trait-like dispositional mindfulness is modifiable and can be increased with practice (e.g., Baer, 2003).

In research and clinical settings, mindfulness may be practiced as part of multiple week or month interventions, or as an induction by having individuals practice a brief mindfulness activity. Month long mindfulness-based interventions have been associated with decreases in 
distress, anxiety, depression, and stress in university students (Breedvelt et al., 2019; Galante et al., 2016; Hindman, Glass, Arnkoff, \& Maron, 2015). However, the interventions used in some of these studies do not solely focus on mindfulness activities but include stress reduction strategies as well, which hinders the ability to isolate the specific advantages of mindfulness. Brief mindfulness inductions are associated with a significant reduction of individuals' reactivity to psychological stress in university students (Creswell, Pacilio, Lindsay, \& Brown, 2014). Therefore, this type of activity might be easier for students to include in their daily lives compared to lengthier interventions while still allowing them to experience some mental health benefits.

Several studies have shown that dispositional mindfulness levels tend to be much lower in individuals who engage in NSSI as well as be associated with negative out- comes such as depression, anxiety, and lower coping self-efficacy (Caltabiano \& Martin, 2017; Garisch \& Wilson, 2015; Heath, Carsley, De Riggi, Mills, \& Mettler, 2016; Heath, Joly, \& Carsley, 2016). As such, improving dispositional mindfulness through practice in those who engage in selfinjury could lead to healthier coping (Caltabiano \& Martin, 2017; Wupperman, Fickling, Klemanski, Berking, \& Whitman, 2013). Studies have examined the use of mindfulness interventions with participants with a history of NSSI engagement in the context of Dialectical Behavior Therapy (DBT; Linehan, 1993), which is a form of cognitive behavior therapy used in the treatment of NSSI engagement (e.g., Ben-Porath, Wisniewski, \& Warren, 2009; KlaininYobas, Cho, \& Creedy, 2012). However, no studies to the authors' knowledge have examined the use of brief mindful- ness inductions with individuals who have a history of NSSI engagement despite being more accessible than traditional forms of therapy. 
This is an Accepted Manuscript of an article published by Taylor \& Francis in 'Archives of Suicide Research' on 2020-11-01, available online: https://www.tandfonline.com/10.1080/13811118.2020.1841052.

on state mindfulness (objective 1) and perceived stress (objective 2) following a stress induction task with university students with a history of NSSI engagement com- pared to those without. It was expected that state mindfulness would be higher and the stress response would be lower following a stress induction task for those who took part in the brief mindfulness activity compared to those in the control condition. Furthermore, no hypotheses were made in regards to the relative differences in response to the mindfulness activity between the NSSI and noNSSI groups as this was explora- tory in the absence of previous research in the area.

\section{METHOD}

\section{Participants}

The University's Research Ethics Review Board approved the study prior to any data collection. Participants were recruited from a database of undergraduate students at a large urban university who participated in a previous non-experimental study on stress and coping with additional participants recruited using advertisements on campus and student social media pages. Inclusion criteria was limited to being at least 18 years of age and a current undergraduate student. Due to inability to recruit a sufficient number of males with a history of NSSI engagement, the final sample was overwhelmingly female and males were excluded from analyses $(n=23)$. Another 5 participants were excluded due to only completing the online portion of the study as detailed in the procedure below. A total of 144 female university students $\left(M_{\text {age }}=20.17\right.$ years, $\left.S D=1.98\right)$ participated in the present study. Approximately half of the sample (54.9\%; $n=79)$ identified as Caucasian, $30.6 \%$ identified as Asian $(n=44), 6.3 \%$ identified as mixed $(n=9), 2.8 \%$ identified as Black $(n=4), 2.1 \%$ identified as Latin American/ Hispanic $(n=3), 2.1 \%$ identified as Middle Eastern $(n=3), 0.7 \%$ identified as First Nations $(n=$ 1), and one participant did not identify their racial or ethnic background $(0.7 \%)$. The final sample 
consisted of 57 women $\left(M_{\text {age }}=20.09\right.$ years, $\left.S D=2.05\right)$ who reported NSSI engagement at least once in their life and 87 women $\left(M_{\text {age }}=20.22\right.$ years, $\left.S D=1.94\right)$ who did not report any NSSI engagement. $70.9 \%$ of those reporting engaging in NSSI had done so over 100 times and 70.2\% within the last two years.

\section{Measures}

\section{Inventory of Statements about Self-Injury (ISAS)}

To determine whether participants had a lifetime history of NSSI engagement, participants were asked the following question: "Have you ever engaged in self-injury without wanting to die (e.g., self-cutting, self-hitting, burning, bruising, scratching, etc.)". Those who indicated NSSI engagement at any point in their life were asked to complete the Inventory of Statements about Self-Injury (ISAS; Klonsky \& Glenn, 2009) which includes a measure of lifetime frequency of 12 different NSSI behaviors as well as their functions. The ISAS demonstrated excellent internal consistency $(\alpha=.79)$ in the present study.

\section{State Mindfulness Scale}

State mindfulness was assessed using the State Mindfulness Scale (SMS; Tanay \& Bernstein, 2013). The scale is composed of 21 items such as "I was aware of different emotions that arose in me". Participants rated how well each statement describes their experience from 1 (Not at all) to 5 (Very well). The SMS demonstrated excellent reliability in the present sample $(\alpha$ $=.93)$.

\section{Visual Analogue Scale}

State stress was assessed using a visual analogue scale (VAS; Lesage, Berjot, \& Deschamps, 2012). On a single item, participants indicate how stressed they feel on an unmarked ruler with anchors labeled as 1 (Not stressed at all) to 100 (As bad as it could be). The scale 
This is an Accepted Manuscript of an article published by Taylor \& Francis in 'Archives of Suicide Research' on 2020-11-01, available online: https:/www.tandfonline.com/10.1080/13811118.2020.1841052.

provides a single score between 1 and 100 depending on the participant's response.

\section{Procedure}

Prior to coming to the laboratory, each participant completed an online questionnaire that included demographic items and the ISAS (see Figure 1 for flowchart of procedure). Data from the online questionnaire was reviewed for a history of NSSI. Using random assignment and a single-blind method, roughly half of those with NSSI and those without NSSI were placed into the mindfulness activity condition (MIND) or the control task condition (LETTER). Once participants arrived at the laboratory, they completed pre-intervention assessments of the SMS and VAS. This was followed by the task to which they were randomly assigned.

Participants ( $n=68 ; 26$ NSSI) in the MIND condition completed a 10 min guided body scan recording available online that was selected by the researchers before the stress induction task (Mindfulness Meditation, 2018). The body scan meditation has been shown to induce a state of mindfulness (e.g., Carmody \& Baer, 2008) and is commonly used with individuals who have no previous experience with mindfulness activities. Participants ( $n=76 ; 31 \mathrm{NSSI})$ in the LETTER condition completed a 10 min letter task that has been piloted and shown not to affect mindfulness or anxiety levels (i.e., serves well as a neutral attention task) in a previous study (e.g., Carsley \& Heath, 2019). Participants in the LETTER condition were provided with a letter and number matrix activity that consisted of a printed document which contained 100 various letters, numbers, and symbols and a grid of 100 boxes. The goal of the activity was to place all of the characters in the grid in any order they wanted. Participants were asked to engage with the task for $10 \mathrm{~min}$.

Following the completion of their respective tasks, participants in both the MIND and LETTER groups underwent a stress induction task using the Stroop color-word interference 
task (Stroop, 1935). No measures were completed between the activity and the Stroop task. The task requires participants to read aloud a first list of colors in black ink followed by a second list of colors that is printed in their respective color. Finally, participants are presented with a third list that includes color words printed in different colors from the word itself and are asked to name the color of the ink. This test is a widely used cognitive task that has been shown to be useful as a lab stressor when executed under time constraints (Karthikeyan, Murugappan, \& Yaacob, 2014; Skoluda et al., 2015; Tulen, Moleman, van Steenis, \& Boomsma, 1989). This task was deemed most appropriate given the present sample of participants who may be sensitive to other stress inducement methods that involve physical pain (e.g., the cold pressor test). The procedure used for this task was the one detailed by Skoluda et al. (2015). Once the stress induction task was completed, all participants completed the post-intervention SMS and VAS measures.

Finally, participants were informed by the research assistant that the experimental part of the project was finished and were asked to watch predetermined funny videos as well as complete repeated measures of state stress until their stress levels returned to or were less than their pre-intervention levels. They were then provided with a debrief form and compensated.

\section{Statistical Analysis}

To examine the proposed objectives, a three-way mixed ANOVA was selected to determine whether there was an interaction effect between group, condition, and time. G*Power (Faul, Erdfelder, Lang, \& Buchner, 2007) was used to run an a priori power analysis using a medium effect size and a desired power of .80 to determine the minimum required sample size $(n$ $=68)$.

All of the following analyses were conducted on SPSS version 24. A missing values 
This is an Accepted Manuscript of an article published by Taylor \& Francis in 'Archives of Suicide Research' on 2020-11-01, available online: https:/www.tandfonline.com/10.1080/13811118.2020.1841052.

analysis was conducted and revealed data was missing completely at random (MCAR) given that less than 5\% of data points were missing per variable (Tabachnick \& Fidell, 2013). Missing values were imputed using the Expectation Maximization (EM) imputation method for SPSS. There were no violations of normality identified.

\section{RESULTS}

\section{Preliminary Descriptives}

The most commonly reported methods of self-injury were cutting with $41.4 \%$ of the NSSI group stating that this was their main form of self-injury $(n=24)$, and severe scratching, which was endorsed as a main form of self-harm by $25.9 \%$ of the NSSI group $(n=15)$. Most individuals reported starting to engage in self-injury between the ages of 12 and $15(63.8 \%, n=$ 36).

\section{Preliminary Analyses}

Two one-way ANOVAs examining differences in pre-intervention stress were conducted to determine group equivalency (see Table 1 for means and standard deviations). The first oneway ANOVA determined that pre-intervention stress did not statistically significantly differ within the NSSI group in the MIND condition and LETTER condition, $F(1,56)=.82, p=.37$. The second one-way ANOVA demonstrated that pre-intervention stress also did not statistically significantly differ between individuals in the no-NSSI group in the MIND and LETTER condition, $F(1,86)=.20, p=.66$. Two additional one-way ANOVAs examining differences in pre-intervention state mindfulness were conducted for the same reasons and determined that the NSSI group, $F(1,56)=1.65, p=.204$, and the no-NSSI group, $F(1,86)=.01, p=.94$, did not statistically significantly differ from one another. Two additional one-way ANOVAs were conducted to determine baseline differences in state mindfulness and stress between the NSSI 
and no-NSSI groups. The first did not reveal significant differences between both groups on state mindfulness, $F(1,86)=.76, p=.39$. The second demonstrated significant differences in stress, $F(1,86)=6.58, p=.01$, whereby individuals who indicated a history of NSSI reported higher baseline stress than those who did not.

\section{Objective 1}

The purpose of objective 1 was to compare differences between groups (NSSI/no-NSSI) and conditions (mindfulness/no-MF) in terms of state mindfulness following the stress induction. A 3-way interaction between group (NSSI, no-NSSI), condition (MF/ no-MF), and time (baseline/follow-up) was hypothesized (H1). A three-way mixed ANOVA (Group X Condition $\mathrm{X}$ Time) was conducted to examine whether a brief mindfulness technique was effective at increasing mindfulness following a stress induction and whether the effects of this task differed between individuals in the different groups (NSSI/no-NSSI) and conditions (MF/no-MF) in order to determine whether the technique was successful at eliciting a state of mindfulness.

There was no statistically significant three-way interaction between group (NSSI/noNSSI), condition (MF/no-MF), and time (baseline/ follow-up), Wilk's $\Lambda=1.00, F(1$, $140)=.58, p=.81, \eta_{p}^{2}=.000$. There was also no statistically significant two-way interaction of time and NSSI group membership, Wilk's $\Lambda=.99, F(1,140)=.67, p=.41, \eta_{p}^{2}=.01$, indicating that state mindfulness levels did not differ as a result of NSSI status alone.

There was a statistically significant two-way interaction of time and condition, Wilk's $\Lambda$ $=.93, F(1,140)=10.70, p=.001, \eta_{p}^{2}=.07$. Follow-up analyses with a Bonferroni-corrected alpha $(p<.025)$ revealed no significant effect of condition on pre-intervention state mindfulness, $F(1,140)=1.27, p=.265$; however, the effect of condition on post-intervention state mindfulness levels was significant, $F(1,140)=8.20, p=.006$. Post-intervention state 
This is an Accepted Manuscript of an article published by Taylor \& Francis in 'Archives of Suicide Research' on 2020-11-01, available online: https:/www.tandfonline.com/10.1080/13811118.2020.1841052.

mindfulness levels were 14.33 units higher in the MIND condition than in the LETTER condition, 95\% CI [4.30, 24.36], $p=.006$. These results revealed that individuals who received the mindfulness activity showed a significant increase in state mindfulness from pre- to postintervention with a medium effect size whereas those who completed the control task did not. This indicates that the mindfulness technique was successful at increasing levels of state mindfulness in both groups. See Figure 2 for mean pre-intervention and post-intervention levels of state mindfulness according to condition.

\section{Objective 2}

The purpose of objective 2 was to compare differences between groups (NSSI/no-NSSI) and conditions (MIND/LETTER) in terms of self-reported stress following the stress induction. A second three-way mixed ANOVA (Group X Condition X Time) was conducted to examine whether a brief mindfulness technique was effective at decreasing stress following a stress induction and whether the effects of this task differed between individuals in the different groups and conditions.

There was no statistically significant 3-way interaction between group, condition, and time, Wilk's $\Lambda=1.00, F(1,140)=.09, p=.77, \eta_{p}^{2}=.001$. There was also no statistically significant two-way interaction of time and NSSI group membership, Wilk's $\Lambda=1.00, F(1,140)$ $=.31, p=.58, \eta_{p}^{2}=.002$, indicating that there were no significant group differences in stress levels from pre- to post- intervention as a result of NSSI status alone.

There was a statistically significant simple two-way interaction of time and condition, Wilk's $\Lambda=.97, F(1,140)=4.21, p=.04, \eta_{p}^{2}=.03$. Follow-up analyses with a Bonferronicorrected alpha $(p<.025)$ revealed no significant effect of condition on pre-intervention stress, $F(1,140)=1.04, p=.311$; however, the effect of condition on post-intervention stress levels was 
significant, $F(1,140)=5.56, p=.02$. Post-intervention stress levels were 9.72 units lower in the MIND condition than in the LETTER condition, 95\%CI $[-17.90,-1.57], p=.02$. Partial etasquared indicated a small to medium effect size. See Figure 3 for mean pre- and postintervention levels of stress according to condition.

\section{DISCUSSION}

The goal of the present study was to determine the effectiveness of a brief mindfulness induction at increasing mindfulness levels and decreasing stress levels in individuals with a history of NSSI engagement compared to those without NSSI, following a stress induction. In terms of state mindfulness, all individuals who were in the mindfulness condition, regardless of NSSI status, showed a significant increase in state mindfulness following the mindfulness induction compared to those who were in the control task condition. Thus, this single 10 min body scan recording successfully elicited a state of mindfulness in university students regardless of NSSI status. To the author's knowledge, no research thus far has examined the effectiveness of such a short activity in those with a history of NSSI engagement.

Moreover, not only was this technique effective at eliciting a state of mindfulness in both groups of females, but it was equally effective in both groups demonstrating its versatility. This finding has clear clinical implications given the support it provides for one simple strategy that is easily accessible to mental health professionals. Additionally, the body scan could be beneficial in group therapy sessions that may include both individuals with and without a history of NSSI engagement. Future research should deter- mine whether continued use of these kinds of techniques can gradually increase dispositional mindfulness and its associated benefits such as greater psychosocial well- being, reduced depressive symptomatology, and reduced perceived stress (e.g., Branstrom, Duncan, \& Moskowitz, 2011; Calvete, Morea, \& Orue, 2019; 
This is an Accepted Manuscript of an article published by Taylor \& Francis in 'Archives of Suicide Research' on 2020-11-01, available online: https:/www.tandfonline.com/10.1080/13811118.2020.1841052.

Dixon \& Overall, 2016; Kong, Wang, Song, \& Liu, 2016; Lundwall, Fairborn, QuinonesCamacho, Estep, \& Davis, 2019).

Given that individuals who engage in NSSI report higher levels of stress (e.g., Kiekens et al., 2015), the similar decrease in stress between those with and without a history of NSSI engagement is especially interesting. This emphasizes that participating in a $10 \mathrm{~min}$ mindfulness activity is sufficient to decrease stress levels of individuals with a history of NSSI engagement and those without in very similar ways. Although Gratz (2007) and other researchers (e.g., Hasking et al., 2010) demonstrate that those with a history of NSSI engagement are emotionally dysregulated, the present study found that their regulatory response to a mindfulness activity is similar to those without such a history. Although recency of NSSI engagement was not taken into account in the present study, an interesting next step would be to compare the effect of a similar mindfulness activity in those with a recent history of NSSI engagement and those with a more distant history.

Stress can be seen as a catalyst for engagement in NSSI given that research has shown that emotion regulation, managing distress, and affect regulation are the most commonly cited reasons for engaging in NSSI (Klonsky, 2011; Victor, Styer, \& Washburn, 2016). Therefore, reducing the intensity of stress experienced following stressful experiences in individuals who engage in NSSI might help reduce the need to engage in NSSI. Overtime, this could perhaps relieve some of the intensity which may trigger individuals to engage in NSSI.

Overall, these results demonstrate that individuals with a history of NSSI engagement do not differ from those without such a history in terms of their response to a brief mindfulness induction both in their state mindfulness and stress levels. This indicates that mindfulness inductions such as the one in the present study do not need to be tail- ored to either group in 
order to be effective, thus highlighting the potential utility of this kind of activity in settings such as universities or in communities with limited resources available.

However, a limitation of the present study is that the sample was exclusively female which is a common drawback of much NSSI literature (Cipriano, Cella, \& Cotrufo, 2017). Additionally, research on gender differences in responses to mindfulness treat- ments or activities are mixed (e.g., Bodenlos, Strang, Gray-Bauer, Faherty, \& Ashdown, 2017) suggesting that more research is needed to clarify whether gender might play a role in terms of responsiveness or even acceptability of mindfulness activities. Additionally, anyone with a history of NSSI engagement no matter the recency was included in the NSSI group, which is a limitation of the group classifications in the pre- sent study.

Although this study attempted to induce stress in participants by using the Stroop task which has been shown to reliably induce a state of stress (Skoluda et al., 2015; Stroop, 1935), a quick examination of participants' stress levels in the LETTER condition indicate that stress did not increase. This reveals that the Stroop task was not effective at inducing stress in the present sample. Individuals with a lifetime history of NSSI engagement have been found to be hyperreactive to stress, therefore, for ethical reasons the Stroop task was selected as a milder stress induction task compared to others such as the Trier Social Stress Test (TSST; Kirschbaum, Pirke, \& Hellhammer, 1993). However, according to Renaud and Blondin (1997), the pace at which the Stroop task is completed as well as the amount of trials completed might affect participant stress levels. Applying this to the use of the task in the present study, participants, although asked to move through the task as quickly as possible, could pace themselves as desired and only completed one round of the task. This could have potentially interfered with the stress-inducing ability. Not only should the present study be replicated using a 
This is an Accepted Manuscript of an article published by Taylor \& Francis in 'Archives of Suicide Research' on 2020-11-01, available online: https:/www.tandfonline.com/10.1080/13811118.2020.1841052.

different stress induction task, but taking into account daily life stressors and whether mindfulness activities alleviate some of that stress as well would be an important next step. In conclusion, the present study was one of the first to find that a brief mindfulness activity is not merely effective for those without a history of NSSI engagement and can therefore be recommended for a wide range of university students for their state mindfulness increasing and stress decreasing benefits. This is particularly interesting given that these students may have varying abilities in terms of emotion regulation but still responded to the mindfulness induction similarly. It is important to note that benefits were reported despite the brevity of the activity highlighting that limited time should not be a barrier to experiencing these benefits. Brief mindfulness activities such as the one used in the present study are becoming increasingly available online and through smartphone applications; therefore, the importance of examining the benefits is imperative. 


\section{REFERENCES}

Baer, R. A. (2003). Mindfulness training as a clinical intervention: A conceptual and empirical review. Clinical Psychology: Science and Practice, 10(2), 125-143.

Ben-Porath, D. D., Wisniewski, L., \& Warren, M. (2009). Differential treatment response for eating disordered patients with and without a comorbid borderline personality diagnosis using a dialectical behavior therapy (DBT)-informed approach. Eating Disorders, 17(3), $225-241$.

Bishop, S. R., Lau, M., Shapiro, S., Carlson, L., Anderson, N. D., Carmody, J., ... Devins, G. (2006). Mindfulness: A proposed operational definition. Clinical Psychology: Science and Practice, 11(3), 230-241.

Bodenlos, J. S., Strang, K., Gray-Bauer, R., Faherty, A., \& Ashdown, B. K. (2017). Male representation in randomized clinical trials of mindfulness-based therapies. Mindfulness, 8(2), 259-265. Br€anstro€m, R., Duncan, L. G., \& Moskowitz, J. T. (2011). The association between dispositional mindfulness, psychological well-being, and perceived health in a Swedish population-based sample. British Journal of Health Psychology, 16(2), 300-316. doi: $\underline{10.1348 / 135910710 \times 501683}$

Breedvelt, J. J. F., Amanvermez, Y., Harrer, M., Karyotaki, E., Gilbody, S., Bockting, C. L. H., ...Ebert, D. D. (2019). The effects of meditation, yoga and mindfulness on depression, anxiety and stress in tertiary education students: A meta-analysis. Frontiers in Psychiatry, 10, 193.

Brown, K. W., \& Ryan, R. M. (2003). The benefits of being present: Mindfulness and its role in psychological well-being. Journal of Personality and Social Psychology, 84(4), 822-848. doi: $10 . \underline{1037 / 0022-3514.84 .4 .822}$ 
This is an Accepted Manuscript of an article published by Taylor \& Francis in 'Archives of Suicide Research' on 2020-11-01, available online: https:/www.tandfonline.com/10.1080/13811118.2020.1841052.

Caltabiano, G., \& Martin, G. (2017). Mindless suffering: The relationship between mindfulness and non-suicidal self-injury. Mindfulness, 8(3), 788-796.

Calvete, E., Morea, A., \& Orue, I. (2019). The role of dispositional mindfulness in the longitudinal associations between stressors, maladaptive schemas, and depressive symptoms in adolescents. Mindfulness, 10(3), 547-558. doi:10.1007/s12671-018-1000-6

Carmody, J., \& Baer, R. A. (2008). Relationships between mindfulness practice and levels of mindfulness, medical and psychological symptoms and well-being in a mindfulnessbased stress reduction program. Journal of Behavioral Medicine, 31(1), 23-33. doi: $\underline{10.1007 / s 10865-\underline{007-9130-7}}$

Carsley, D., \& Heath, N. L. (2019). Effectiveness of mindfulness-based coloring for university students' test anxiety. Journal of American College Health, 68(5), 518-527.

Cash, M., \& Whittingham, K. (2010). What facets of mindfulness contribute to psychological well-being and depressive, anxious, and stress-related symptomatology? Mindfulness, 1(3), 177-182.

Cipriano, A., Cella, S., \& Cotrufo, P. (2017). Nonsuicidal self-injury: A systematic review. Frontiers in Psychology, 8, 1946.

Creswell, J. D., \& Lindsay, E. K. (2014). How does mindfulness training affect health? A mindful- ness stress buffering account. Current Directions in Psychological Science, 23(6), 401-407. doi: $\underline{10.1177 / 0963721414547415}$

Creswell, J. D., Pacilio, L. E., Lindsay, E. K., \& Brown, K. W. (2014). Brief mindfulness meditation training alters psychological and neuroendocrine responses to social evaluative stress. Psychoneuroendocrinology, 44, 1-12. doi:10.1016/j.psyneuen.2014.02.007 
Deliberto, T. L., \& Nock, M. K. (2008). An exploratory study of correlates, onset, and offset of non-suicidal self-injury. Archives of Suicide Research: Official Journal of the International Academy for Suicide Research, 12(3), 219-231. doi: $\underline{10.1080 / 13811110802101096}$

Dixon, H. C., \& Overall, N. C. (2016). Dispositional mindfulness attenuates the link between daily stress and depressed mood. Journal of Social and Clinical Psychology, 35(3), 255268. doi: $\underline{10.1521 / \mathrm{jscp} .2016 .35 .3 .255}$

Faul, F., Erdfelder, E., Lang, A., \& Buchner, A. (2007). G*Power 3: A flexible statistical power analysis program for the social, behavioral, and biomedical sciences. Behavior Research Methods, 39(2), 175-191. doi:10.3758/bf03193146

Fliege, H., Kocalevent, R.-D., Walter, O. B., Beck, S., Gratz, K. L., Gutierrez, P. M., \& Klapp, B. F. (2006). Three assessment tools for deliberate self-harm and suicide behavior: Evaluation and psychopathological correlates. Journal of Psychosomatic Research, 61(1), 113-121. doi:10. 1016/j.jpsychores.2005.10.006

Galante, J., Dufour, G., Benton, A., Howarth, E., Vainre, M., Croudace, T. J., ... Jones, P. B. (2016). Protocol for the Mindful Student Study: A randomised controlled trial of the provision of a mindfulness intervention to support university students' well-being and resilience to stress. BMJ Open, 6(11), e012300.

Garisch, J. A., \& Wilson, M. S. (2015). Prevalence, correlates, and prospective predictors of nonsuicidal self-injury among New Zealand adolescents: Cross-sectional and longitudinal survey data. Child and Adolescent Psychiatry and Mental Health, 9(1), 28. doi: $\underline{10.1186 / s 13034-015-\underline{0055-6}}$

Gratz, K. L. (2007). Targeting emotion dysregulation in the treatment of self-injury. Journal of 
This is an Accepted Manuscript of an article published by Taylor \& Francis in 'Archives of Suicide Research' on 2020-11-01, available online: https:/www.tandfonline.com/10.1080/13811118.2020.1841052.

Clinical Psychology, 63(11), 1091-1103.

Hamza, C. A., \& Willoughby, T. (2014). A longitudinal person-centered examination of nonsuicidal self-injury among university students. Journal of Youth and Adolescence, 43(4), 671-685. doi:10.1007/s10964-013-9991-8

Hasking, P. A., Coric, S. J., Swannell, S., Martin, G., Thompson, H. K., \& Frost, A. D. (2010). Brief report: Emotion regulation and coping as moderators in the relationship between person- ality and self-injury. Journal of Adolescence, 33(5), 767-773. doi: $\underline{10.1016 / j . a d o l e s c e n c e .2009 .12 .} \underline{006}$

Hasking, P., Momeni, R., Swannell, S., \& Chia, S. (2008). The nature and extent of non-suicidal self-injury in a non-clinical sample of young adults. Archives of Suicide Research, 12(3), 208-218. doi: $\underline{10.1080 / 13811110802100957}$

Hayes, A. M., \& Feldman, G. (2004). Clarifying the construct of mindfulness in the context of emotion regulation and the process of change in therapy. Clinical Psychology: Science and Practice, 11(3), 255-262.

Heath, N. L., Carsley, D., De Riggi, M. E., Mills, D., \& Mettler, J. (2016). The relationship between mindfulness, depressive symptoms, and non-suicidal self-injury amongst adolescents. Archives of Suicide Research: Official Journal of the International Academy for Suicide Research, 20(4), 635-649. doi:10.1080/13811118.2016.1162243

Heath, N. L., Joly, M., \& Carsley, D. (2016). Coping self-efficacy and mindfulness in nonsuicidal self-injury. Mindfulness, 7(5), 1132-1141. doi:10.1007/s12671-016-0555-3

Hilt, L. M., Nock, M. K., Lloyd-Richardson, E. E., \& Prinstein, M. J. (2008). Longitudinal study of nonsuicidal self-injury among young adolescents: Rates, correlates, and preliminary test of an interpersonal model. The Journal of Early Adolescence, 28(3), 455-469. 
doi: $\underline{10.1177 /} \underline{0272431608316604}$

Hindman, R. K., Glass, C. R., Arnkoff, D. B., \& Maron, D. D. (2015). A comparison of formal and informal mindfulness programs for stress reduction in university students. Mindfulness, 6(4), 873-884.

International Society for the Study of Self-Injury (ISSS). (2007). Definitional issues surrounding our understanding of self-injury. Paper presented at Conference proceedings from the annual meeting.

Kabat-Zinn, J. K. (1994). Wherever you go, there you are: Mindfulness meditation in everyday life. New York, NY: Hyperion.

Karthikeyan, P., Murugappan, M., \& Yaacob, S. (2014). Analysis of Stroop Color Word Testbased human stress detection using electrocardiography and heart rate variability signals. Arabian Journal for Science and Engineering, 39(3), 1835-1847.

Kiekens, G., Bruffaerts, R., Nock, M. K., Van de Ven, M., Witteman, C., Mortier, P., ... Claes, L. (2015). Non-suicidal self-injury among Dutch and Belgian adolescents: Personality, stress, and coping. European Psychiatry: The Journal of the Association of European Psychiatrists, 30(6), 743-749.

Kiken, L. G., Garland, E. L., Bluth, K., Palsson, O. S., \& Gaylord, S. A. (2015). From a state to a trait: Trajectories of state mindfulness in meditation during intervention predict changes in trait mindfulness. Personality and Individual Differences, 81, 41-46. doi:10.1016/j.paid.2014.12. $\underline{044}$

Kirschbaum, C., Pirke, K. M., \& Hellhammer, D. H. (1993). The 'Trier Social Stress Test'-a tool for investigating psychobiological stress responses in a laboratory setting. Neuropsychobiology, 28(1-2), 76-81. doi:10.1159/000119004 
This is an Accepted Manuscript of an article published by Taylor \& Francis in 'Archives of Suicide Research' on 2020-11-01, available online: https:/www.tandfonline.com/10.1080/13811118.2020.1841052.

Klainin-Yobas, P., Cho, M. A. A., \& Creedy, D. (2012). Efficacy of mindfulness-based interventions on depressive symptoms among people with mental disorders: A metaanalysis. International Journal of Nursing Studies, 49(1), 109-121. doi: 10.1016/j.ijnurstu.2011.08.014

Klonsky, E. D. (2007). The functions of deliberate self-injury: A review of the evidence. Clinical Psychology Review, 27(2), 226-239.

Klonsky, E. D. (2011). Non-suicidal self-injury in United States adults: Prevalence, sociodemographics, topography and functions. Psychological Medicine, 41(9), 1981-1986.

Klonsky, E. D., \& Glenn, C. R. (2009). Assessing the functions of non-suicidal self-injury: Psychometric properties of the Inventory of Statements About Self-injury (ISAS). Journal of Psychopathology and Behavioral Assessment, 31(3), 215-219. doi:10.1007/s10862$\underline{008-9107-\mathrm{Z}}$

Klonsky, E. D., \& Muehlenkamp, J. J. (2007). Self-injury: A research review for the practitioner. Journal of Clinical Psychology, 63(11), 1045-1056. doi: 10.1002/jclp.20412

Kong, F., Wang, X., Song, Y., \& Liu, J. (2016). Brain regions involved in dispositional mindfulness during resting state and their relation with well-being. Social Neuroscience, 11(4), 331-343. doi:10.1080/17470919.2015.1092469

Lau, M. A., Bishop, S. R., Segal, Z. V., Buis, T., Anderson, N. D., Carlson, L., .. Devins, G. (2006). The Toronto mindfulness scale: Development and validation. Journal of Clinical Psychology, 62(12), 1445-1467.

Lesage, F. X., Berjot, S., \& Deschamps, F. (2012). Clinical stress assessment using a visual analogue scale. Occupational Medicine, 62(8), 600-605. doi: $\underline{10.1093 / \text { occmed/kqs140 }}$ 
Linehan, M. (1993). Skills training manual for treating borderline personality disorder (Vol. 29). New York, NY: Guilford Press.

Lundwall, C., Fairborn, S., Quinones-Camacho, L., Estep, J., \& Davis, E. (2019). Self-regulation mechanisms explain how dispositional mindfulness promotes well-being. Journal of Positive Psychology and Wellbeing, 3(2), 153-164.

Mindfulness Meditation. (2018, May 11). 10 minute guided body scan meditation for beginners [VideoFile]. Retrieved from: https://www.youtube.com/watch?v=vmopsvmenjy.

Nilsson, H., \& Kazemi, A. (2016). Reconciling and thematizing definitions of mindfulness: The big five of mindfulness. Review of General Psychology, 20(2), 183-193. doi:10.1037/gpr0000074 Nock, M. K., \& Prinstein, M. J. (2004). A functional approach to the assessment of self-mutilative behavior. Journal of Consulting and Clinical Psychology, 72(5), 885-890. doi:10.1037/0022- 006X.72.5.885

Reichl, C., Heyer, A., Brunner, R., Parzer, P., Vo€lker, J. M., Resch, F., \& Kaess, M. (2016). Hypothalamic-pituitary-adrenal axis, childhood adversity and adolescent nonsuicidal self- injury. Psychoneuroendocrinology, 74, 203-211. doi:10.1016/j.psyneuen.2016.09.011

Renaud, P., \& Blondin, J. P. (1997). The stress of Stroop performance: Physiological and emotional responses to color-word interference, task pacing, and pacing speed. International Journal of Psychophysiology: Official Journal of the International Organization of Psychophysiology, 27(2), 87-97. doi:10.1016/s0167-8760(97)00049-4

Segal, Z. V., Teasdale, J. D., Williams, J. M., \& Gemar, M. C. (2002). The mindfulness-based cognitive therapy adherence scale: Inter-rater reliability, adherence to protocol and treatment distinctiveness. Clinical Psychology \& Psychotherapy, 9(2), 131-138. 
This is an Accepted Manuscript of an article published by Taylor \& Francis in 'Archives of Suicide Research' on 2020-11-01, available online: https://www.tandfonline.com/10.1080/13811118.2020.1841052.

Serras, A., Saules, K. K., Cranford, J. A., \& Eisenberg, D. (2010). Self-injury, substance use, and associated risk factors in a multi-campus probability sample of college students. Psychology of Addictive Behaviors, 24(1), 119-128. doi:10.1037/a0017210

Skoluda, N., Strahler, J., Schlotz, W., Niederberger, L., Marques, S., Fischer, S., ... Nater, U. M. (2015). Intra-individual psychological and physiological responses to acute laboratory stressors of different intensity. Psychoneuroendocrinology, 51, 227-236.

Stroop, J. R. (1935). The basis of Ligon's theory. The American Journal of Psychology, 47(3), 499-504.

Swannell, S. V., Martin, G. E., Page, A., Hasking, P., \& St John, N. (2014). Prevalence of nonsuicidal self-injury in nonclinical samples: systematic review, meta-analysis and meta-regression. Suicide \& Life-Threatening Behavior, 44(3), 273-303. doi: $\underline{10.1111 / \text { sltb. } 12070}$

Tabachnick, B. G., \& Fidell, L. S. (2013). Using multivariate statistics (6th ed.). Colorado, DE: Allyn \& Bacon/Pearson Education.

Tanay, G., \& Bernstein, A. (2013). State Mindfulness Scale (SMS): Development and initial validation. Psychological Assessment, 25(4), 1286-1299. doi:10.1037/a0034044

Tulen, J. H. M., Moleman, P., Van Steenis, H. G., \& Boomsma, F. (1989). Characterization of stress reactions to the Stroop Color Word Test. Pharmacology Biochemistry and Behavior, 32(1), 9-15. doi:10.1016/0091-3057(89)90204-9

Victor, S. E., Styer, D., \& Washburn, J. J. (2016). Functions of nonsuicidal self-injury (NSSI): Cross-sectional associations with NSSI duration and longitudinal changes over time and following treatment. Psychiatry Research, 241, 83-90.

Whitlock, J., Muehlenkamp, J., Purington, A., Eckenrode, J., Barreira, P., Baral Abrams, G., ...\& 
Knox, K. (2011). Nonsuicidal self-injury in a college population: General trends and sex differences. Journal of American College Health, 59(8), 691 698.doi: $\underline{10.1080 / 07448481.2010 .529626}$

Wupperman, P., Fickling, M., Klemanski, D. H., Berking, M., \& Whitman, J. B. (2013). Borderline personality features and harmful dysregulated behavior: The mediational effect of mindfulness. Journal of Clinical Psychology, 69(9), 903-911. doi:10.1002/jclp.21969 
This is an Accepted Manuscript of an article published by Taylor \& Francis in 'Archives of Suicide Research' on 2020-11-01, available online: https://www.tandfonline.com/10.1080/13811118.2020.1841052.

\section{Tables and Figures}

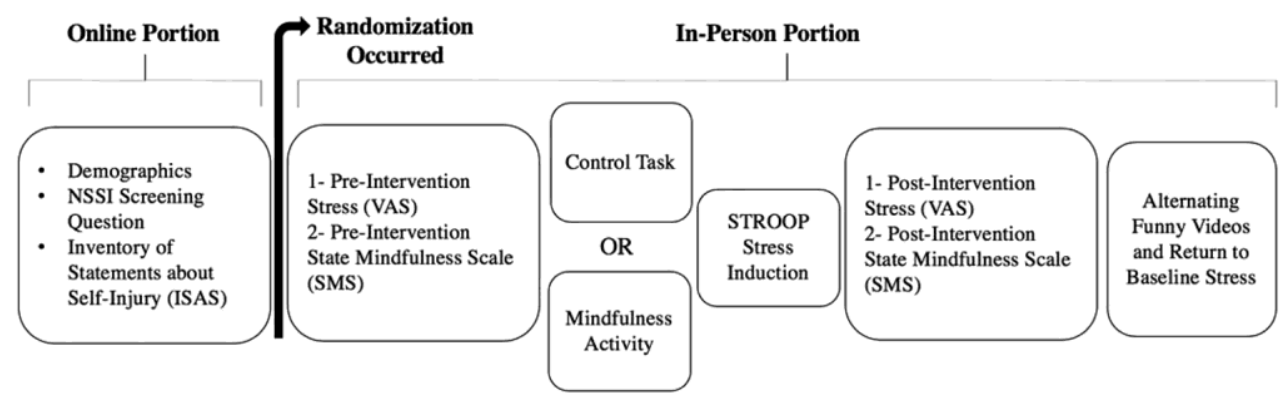

Distributed

One-Week Prior to In-

Person Portion

FIGURE 1. Flowchart of study procedure and measures and tasks completed.

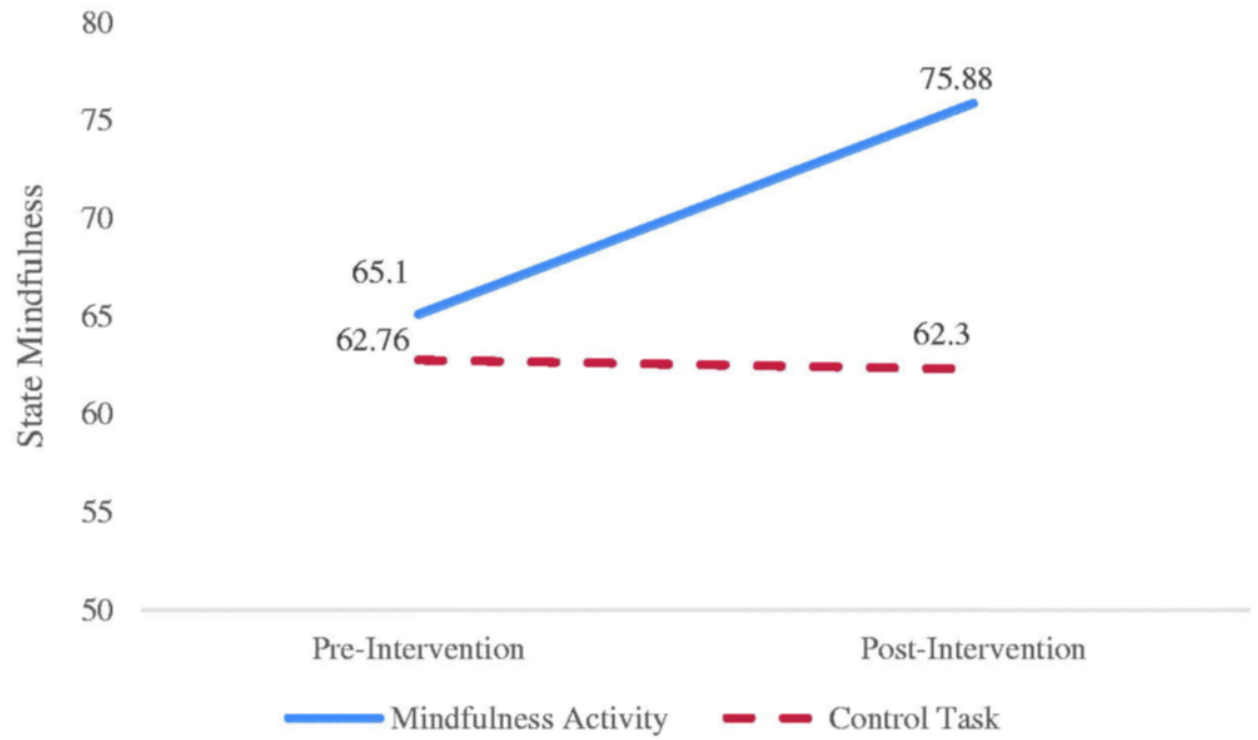

FIGURE 2. Mean pre- and post-intervention levels of state mindfulness according to mindfulness or control task condition. 


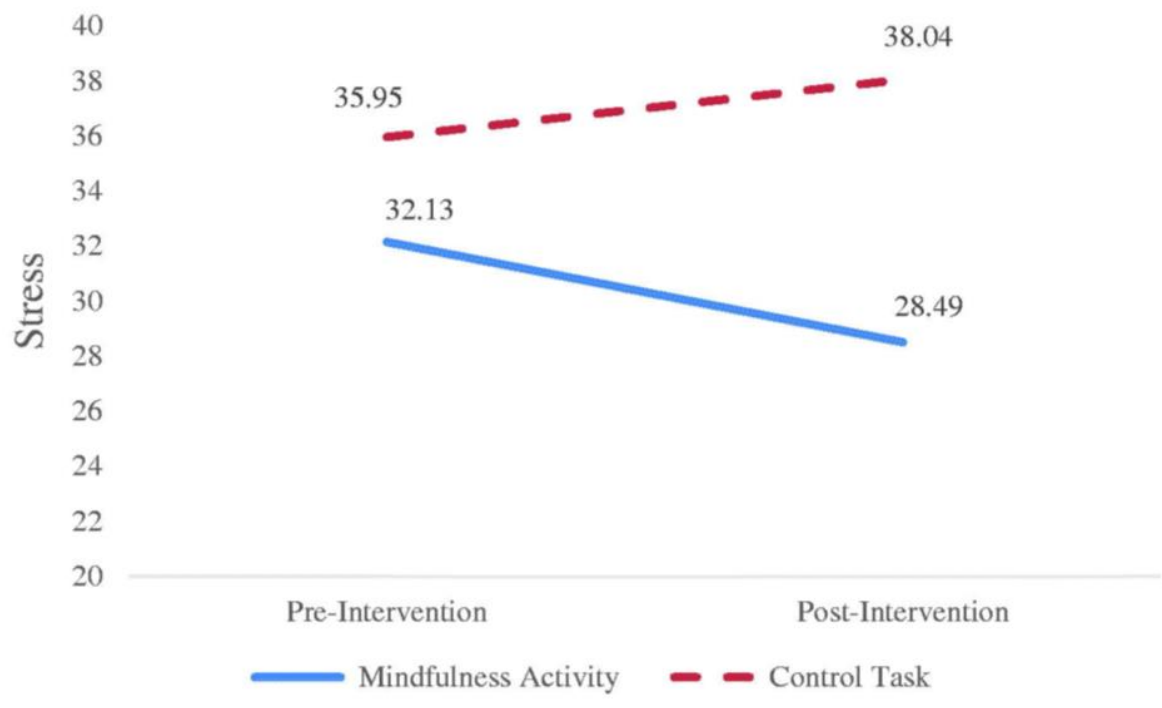

FIGURE 3. Mean pre- and post-intervention levels of stress according to mindfulness or control task condition. 
This is an Accepted Manuscript of an article published by Taylor \& Francis in 'Archives of Suicide Research' on 2020-11-01, available online: https://www.tandfonline.com/10.1080/13811118.2020.1841052.

TABLE 1. Means and standard deviations for state mindfulness and stress for each group and condition at pre- and post-intervention.

NSSI

\begin{tabular}{|c|c|c|c|c|c|c|c|}
\hline \multirow[t]{2}{*}{ Pre-Intervention } & \multicolumn{2}{|c|}{ Post-Intervention } & \multicolumn{3}{|c|}{ Pre-Intervention } & \multicolumn{2}{|c|}{ Post-Intervent } \\
\hline & $\overline{\mathrm{M}} \mathrm{SD}$ & $\overline{\mathrm{M}}$ & SD & $\overline{\mathrm{M}}$ & SD & $\overline{\mathrm{M}}$ & SD \\
\hline \multicolumn{8}{|c|}{ State mindfulness } \\
\hline $\begin{array}{l}\text { Mindfulness } \\
\text { condition }\end{array}$ & 64.8118 .51 & 76.63 & 17.45 & 65.29 & 16.21 & 75.40 & 17.04 \\
\hline $\begin{array}{l}\text { Control task } \\
\text { condition }\end{array}$ & 59.3318 .19 & 62.30 & 20.06 & 65.04 & 14.20 & 64.91 & 15.67 \\
\hline Stress & & & & & & & \\
\hline $\begin{array}{l}\text { Mindfulness } \\
\text { condition }\end{array}$ & 36.7424 .78 & 32.67 & 23.35 & 29.17 & 22.10 & 25.81 & 22.40 \\
\hline $\begin{array}{l}\text { Control task } \\
\text { condition }\end{array}$ & 42.8325 .96 & 43.53 & 23.10 & 31.36 & 23.43 & 34.38 & 26.78 \\
\hline
\end{tabular}

No-NSSI

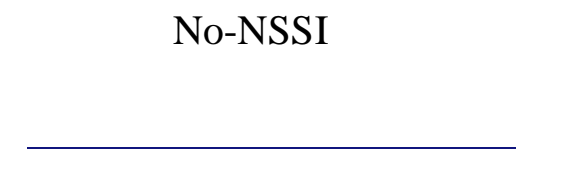

